\title{
Growth of defect-free InAs Nanowires using Pd catalyst
}

\author{
H.Y. Xu ${ }^{1}$, Y.N. Guo ${ }^{1}$, Z.M. Liao ${ }^{1}$, J. Zou ${ }^{1,2^{*}}$, Q. Gao ${ }^{3}$, H.H.Tan ${ }^{3}$, and C. Jagadish ${ }^{3}$ \\ ${ }^{1}$ Materials Engineering and ${ }^{2}$ Centre for Microscopy and Microanalysis, The University of Queensland, St \\ Lucia, 4072, Australia \\ ${ }^{3}$ Department of Electronic Materials Engineering, Research School of Physics and Engineering, The Austra- \\ lian National University, Canberra, ACT 0200, Australia \\ ${ }^{*}$ Corresponding author: j.zou@uq.edu.au
}

\begin{abstract}
To explore the growth mechanism and the effect of non-gold catalysts in growing III-V epitaxial nanowires, InAs nanowires were grown on $\mathrm{GaAs}(111) \mathrm{B}$ substrates using annealed $\mathrm{Pd}$ thin film as catalyst. Through detailed scanning and transmission electron microscopy (SEM/TEM) characterisations, it is found that when the catalyst size is less than 50 $\mathrm{nm}$ (from annealing the Pd thin film), defect-free zinc-blende structured epitaxial InAs nanowires are grown along the $<110>$ directions with four $\{111\}$ sidefacets. The common nanowire/catalyst interface is found to be the unusual $\{113\}$ planes of the nanowires.
\end{abstract}

1 Introduction: In the past decade, significant research efforts have been devoted to the growth of III-V semiconductor nanowires due to its high potential in optoelectronics, sensor and electronic technology $[1,2]$. Au has been widely used to catalyse the growth of III-V epitaxial nanowires on various substrates, while limited studies have been carried out on the growth of III-V nanowires using non$\mathrm{Au}$ catalysts. In this study, we demonstrate the epitaxial growth of InAs nanowires on GaAs (111)B substrates using $\mathrm{Pd}$ as catalyst. The unusual morphological and structural characteristics of InAs nanowires allow the understanding of the effects of $\mathrm{Pd}$ catalysts on the growth of III-V nanowires.

2 Experimental Section: $A \sim 2 \mathrm{~nm}$ thick layer of $\mathrm{Pd}$ film was firstly deposited onto GaAs (111)B substrates using an electron-beam evaporator. The InAs nanowires were grown by metal organic chemical vapor deposition using trimethylindium and arsine with flow rates of $1.16 \times 10^{-5} \mathrm{~mol} / \mathrm{min}$ and $3.35 \times 10^{-5}$ $\mathrm{mol} / \mathrm{min}$, respectively. Prior to the growth, the Pd coated substrate was annealed in the growth chamber at $600^{\circ} \mathrm{C}$ for $10 \mathrm{~min}$ under arsine ambient to desorb surface contaminants and to agglomerate the $\mathrm{Pd}$ thin film into nanoparticles. A growth temperature of $500^{\circ} \mathrm{C}$ was used due to the higher In/Pd eutectic temperature. The grown InAs nanowires and their corresponding catalysts were investigated by SEM (JEOL 7001F/7800F, both operated at $15 \mathrm{kV}$ ) and TEM (Philips F20, operated at $200 \mathrm{kV}$ ) to determine their morphological and structural characteristics.

3 Results and Discussion: Figure 1 shows the SEM images of typical nanowires catalysed by the $\mathrm{Pd}$ thin film. From Figs. $1 \mathrm{~A}$ and $1 \mathrm{~B}$, the nanowires are not perpendicular to the substrate. To determine their growth direction, the nanowire samples were tilted along the
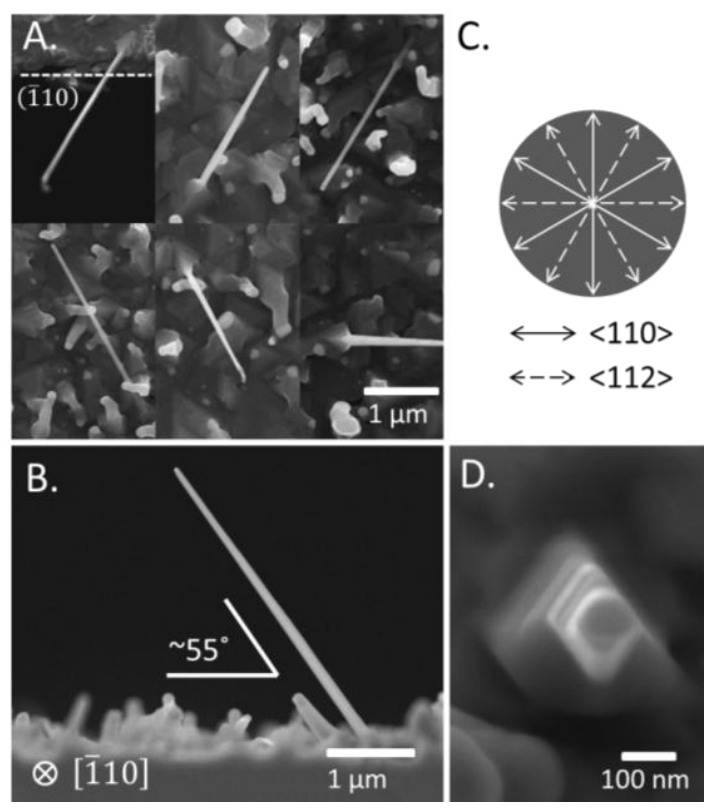

Fig 1 - SEM images of InAs nanowires grown with $\mathrm{Pd}$ catalyst. (A) composite image of the nanowires, $(B)$ side view of the nanowire(s) (C) relationship of projected <110> and <112> directions on (111)B substrate. (D) top view of the asgrown nanowire. 


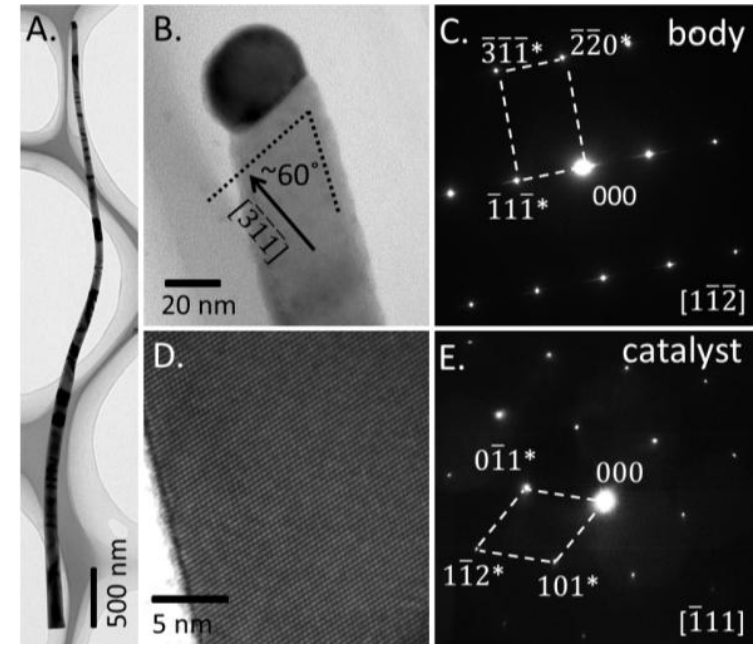

Fig 2 - Typical TEM images of nanowires catalysed by $\mathrm{Pd}$ thin film. (A) an overview, $(B)$ and $(C)$ bright field image and electron diffraction taken at the tip region of the nanowire viewed along the [1]2] zone axis. (D) HRTEM image of the nanowire. (E) electron diffraction of the catalyst taken along [111] zone axis.

$<110>$ axis (refer to Fig. 1C). Figure 1D shows a typical example of a nanowire tilted by $35^{\circ}$ to look down the $\left.<110\right\rangle$ direction, in which the axial direction of the nanowire is parallel to the electron beam. Geometrically, the growth direction is determined as $\langle 110\rangle$, and, simultaneously, the diamond-shaped side-facets of the nanowire are determined as the $\{111\}$ planes.

Figure 2 shows TEM investigations of a typical nanowire, where Fig. 2A shows an entire nanowire. To determine the catalyst/nanowire interface, the nanowire specimen was oriented to the direction where the sharp catalyst/ nanowire interface is seen (as illustrated in Fig. 2B). Figure $2 \mathrm{C}$ is the corresponding electron diffraction and confirms the catalyst/nanowire interface being the un- usual $\{113\}$ plane. Our extensive TEM investigations confirmed that InAs nanowires are defect free (e.g. lack of spots/streaks in the ED shown as Fig. 2D) and the catalyst is the body-centercubic structured InPd phase (refer to Fig. $2 \mathrm{E}$ ). It is believed that our defect-free InAs nanowires benefit from the fact that the growth direction of the nanowire is non-<111> and the nanowire/catalyst interface is non- $\{111\}$ atomic plane. By changing the interface to $\{113\}$, it is difficult to generate lattice defects in the growth front. Moreover, our InAs nanowires have the low-energy $\{111\}$ side-facets, suggesting that the growth of nanowire is dominated by the energy minimization process of the side facets.

4 Conclusion: By using Pd nanoparticles as catalysts, we have successfully grown defect-free InAs nanowires with unusual growth directions, side-facets and nanowire/catalyst interfaces.

\section{Acknowledgements}

This work has been supported by the Australian Research Council. The Australian National Fabrication Facility and Australian Microscopy and Microanalysis Research Facility are gratefully acknowledged for providing access to the facilities used in this research.

\section{References}

[1] H. Xu, Y. Wang, Y. Guo, Z. Liao, Q. Gao, N. Jiang, H.H. Tan, C. Jagadish and J. Zou. "High-Density, Defect-Free, and TaperRestrained Epitaxial GaAs Nanowires Induced from Annealed Au Thin Films", Cryst. Growth Des. 12, pp. 2018-2022, 2012

[2] H. Joyce, J. Wong-Leung, Q. Gao, H.H. Tan and $C$. Jagadish. "Phase Perfection in Zinc Blende and Wurtzite III-V Nanowires Using Basic Growth Parameters", Nano Lett. 10, pp 908-915, 2010. 